\title{
Chiara Giubilaro*
}

\section{Il diritto ad aspirare nelle geografie dei bambini. Una ricerca-azione partecipativa nel quartiere CEP di Palermo}

Parole chiave: aspirazioni urbane, geografia politica dei bambini, Palermo.

Questo lavoro si propone di indagare criticamente il ruolo che le aspirazioni urbane, vale a dire la capacità collettiva di immaginare spazi alternativi per le proprie città, hanno nella costruzione delle geografie quotidiane delle bambine e dei bambini. In linea con i presupposti della Political Geography of Children, bambine e bambini vengono qui considerati come attori socio-spaziali capaci di rinegoziare pratiche e rappresentazioni imposte dagli adulti. Muovendo da queste considerazioni teoriche e dai principi metodologici della ricerca-azione partecipativa, analizzeremo il percorso laboratoriale organizzato con le ragazze e i ragazzi dell'Associazione San Giovanni Apostolo del CEP di Palermo, uno dei quartieri più marginalizzati della città. In particolare, prenderemo in considerazione le attività di photo-walk e di mappatura collettiva condotte nel quartiere e i tentativi di trasformare il campo abbandonato di via Calandrucci da zona di 'disimmaginazione' a luogo di desideri e rivendicazioni per i suoi abitanti più piccoli.

The right to aspire in children's geographies. A participatory action research in Palermo

Keywords: urban aspirations, Political Geography of Children, Palermo.

This article aims at critically investigating how urban aspirations, that is the capacity to collectively imagine and desire alternative spaces for the cities we live in, affect the construction of everyday children's geographies. Drawing on Political Geography of Children, children are here considered as social actors that ceaselessly negotiate spatial practices and representations created and imposed by adults. Combining this theoretical framework with participatory action research methodologies, we analyze the activities carried out with children from San Giovanni Apostolo Onlus Association in the highly

* Università di Palermo, Dipartimento di Architettura, Viale delle Scienze Ed. 14, 90128 Palermo, chiara.giubilaro@unipa.it.

Saggio proposto alla redazione il 9 aprile 2021, accettato il 29 luglio 2021. 


\section{Il diritto ad aspirare nelle geografie dei bambini}

marginalized neighborhood of CEP (Palermo). More specifically, our emphasis is on the techniques of photo-walk and collective mapping along with the efforts of transforming the so-called field of via Calandrucci from a 'disimagination zone' into a space where children and young people can claim their right to aspire.

1. Introduzione. - Nel maggio del 1991 nasce a Fano "La città dei bambini", un progetto ideato e promosso dal pedagogista Francesco Tonucci con l'intento di introdurre un singolare cambio di paradigma nel governo della città ${ }^{1}$. In aperta contrapposizione con la frammentazione degli spazi, la specializzazione delle loro funzioni e il protagonismo dell'automobile che caratterizzano la fruizione della città da parte degli adulti, il progetto mira ad assumere i bambini e le bambine come nuovi parametri di governo e pianificazione dei processi di trasformazione urbana (Baraldi e Maggioni, 2000). In linea con la prospettiva promossa nell'ambito della nuova sociologia dell'infanzia (James et al., 1998; James e Prout, 1990; Saporiti e Sgritta, 1990), "la città dei bambini" riconosce nel bambino un soggetto politico attivo e nella sua peculiare relazione con gli spazi un'opportunità di cambiamento per le amministrazioni delle città italiane. In questi anni, infatti, diversi sono i progetti, le istituzioni e le norme ${ }^{2}$ che cercano di mettere al centro della scena urbana i bambini e le loro esigenze, nel tentativo di tradurre i nuovi approcci introdotti negli studi sull'infanzia e l'adolescenza in concrete esperienze sul territorio. Gli anni Novanta hanno rappresentato in questo senso un decisivo spartiacque nel modo di pensare e praticare le relazioni fra infanzia, politica e città. Se da una parte si è infatti assistito a una proliferazione di progetti promossi da amministrazioni, scuole e università con la specifica finalità di rafforzare la partecipazione di bambini e ragazzi sulla scena politica urbana, dall'altra riflessioni e teorie sui bambini come attori socio-spaziali hanno attraversato campi del sapere differenti, dando luogo a formazioni disciplinari che oggi animano riviste, convegni e dipartimenti.

In questo articolo esploreremo la relazione fra i bambini e la città nella prospettiva della geografia politica dei bambini (Political Geography of Children), un campo di studi che negli ultimi tre decenni ha conquistato l'attenzione dei geografi,

Un ringraziamento particolare agli anonimi revisori, per le preziose critiche che hanno rivolto all'articolo, a Barbara Pizzo e Massimo Massaro, per la cura con cui hanno seguito ogni fase della ricerca, a Maria Antonietta Fazio, per l'ostinazione e l'impegno con cui conduce il lavoro sul territorio, e alle ragazze e ai ragazzi dell'Associazione San Giovanni Apostolo, per la schiettezza e la forza con cui hanno destabilizzato le coordinate del mio modo di fare geografia.

${ }^{1}$ Per ulteriori informazioni sul progetto si rimanda a www.lacittadeibambini.org/progetto.

${ }^{2}$ All'agosto del 1997 risale, per esempio, la legge n. 285/97 "Disposizioni per la promozione di diritti e di opportunità per l'infanzia e l'adolescenza" (Parlamento della Repubblica Italiana, 1997), che istituisce un fondo per il finanziamento di progetti destinati alla tutela e ai diritti dei minori nelle principali città italiane (Baraldi, 2001). 
specie nel mondo anglofono. Muovendo da questo terreno teorico cercheremo di rileggere criticamente l'esperienza di ricerca-azione da me condotta con le ragazze e i ragazzi del CEP di Palermo, un quartiere di edilizia residenziale pubblica fra i più violentemente marginalizzati nella storia e nella geografia della città ${ }^{3}$. In particolare, l'obiettivo è di comprendere quale ruolo possono avere le aspirazioni urbane, vale a dire la capacità collettiva di immaginare spazi alternativi per le proprie città, tanto nel recente dibattito teorico sulla costruzione dell'agency all'interno della geografia politica dei bambini quanto nelle concrete pratiche spaziali adottate dagli attori coinvolti nel corso della ricerca-azione. Nel tentativo di perseguire questi obiettivi, l'articolo sarà così strutturato: la prima parte sarà dedicata a una ricognizione della geografia politica dei bambini con particolare riferimento alle potenzialità e ai limiti che la concettualizzazione dell'agency solleva al suo interno. In questo quadro verrà rideclinata in chiave urbana la categoria di aspirazione proposta dall'antropologo indiano Arjun Appadurai (Appadurai, 2013, trad. it. 2014), nel tentativo di esplorare le possibilità che questa apre per le geografie materiali e immateriali della città. Nella seconda parte riporteremo il quadro teorico ai tortuosi itinerari della ricerca sul terreno e agli interventi che l'hanno accompagnata. Dopo una breve contestualizzazione storica del quartiere CEP e una ricognizione degli assetti metodologici che la letteratura sulla ricerca-azione partecipativa ma soprattutto l'esperienza laboratoriale con le ragazze e i ragazzi del quartiere hanno contribuito a costruire, ripercorreremo i processi di costruzione del laboratorio sulle aspirazioni urbane e i conseguenti tentativi di riqualificazione di un'area in stato di abbandono che hanno variamente incrociato gli itinerari della ricerca.

2. Infanzia, politica e città. - Il 20 novembre 1989 l'Assemblea Generale delle Nazioni Unite approva la "Convention on the Rights of the Child", un documento sottoscritto da tutti i paesi del mondo con la sola eccezione degli Stati Uniti $^{4}$ che ha per oggetto il riconoscimento e la tutela dei diritti dell'infanzia e dell'adolescenza. Nello stesso anno compare sulla rivista Political Geography Quarterly un editoriale dal titolo "Children and Politics" nel quale Peter Taylor auspica l'inclusione dei bambini nell'agenda della geografia politica, in qualità di partecipanti attivi nei processi sociali e politici osservati (Taylor, 1989, p. 5).

Nei trent'anni trascorsi dalla pubblicazione di quell'editoriale e in aperto dialogo con la nuova sociologia dell'infanzia richiamata in apertura, un nuovo campo di studi denominato Children's Geographies si è progressivamente andato affermando sulla scena geografica, combinando insieme approcci e ispirazioni derivati dalla geografia politica con l'attenzione alle differenze e la postura critica propri della

${ }^{3}$ La ricerca qui presentata è stata finanziata dal Programma Sylff della Sylff Association attraverso il Programma di ricerca "Idea-Azione" dell'Istituto di Formazione Politica "Pedro Arrupe".

${ }^{4}$ L'Italia ha sottoscritto la convenzione due anni dopo, nel 1991. 


\section{Il diritto ad aspirare nelle geografie dei bambini}

nuova geografia culturale 5 . Così, mentre in molti paesi europei la convenzione delle Nazioni Unite sui diritti del bambino citata in apertura porta alla proliferazione di progetti e finanziamenti volti a costruire spazi di partecipazione per i minori (Kallio e Häkli, 2011), su riviste e volumi iniziano a comparire contributi che, in questa prima fase, sono legati a doppio filo alle geografie femministe e alle aperture che queste stanno già da alcuni anni introducendo nell'ambito della geografia, specie per quel che riguarda l'inclusione di soggetti tradizionalmente marginalizzati sulla scena sociale, l'attenzione alle differenze e il riconoscimento del carattere politico degli spazi del quotidiano (Staeheli et al., 2013). La discussione sul posto che deve occupare la riflessione sui bambini nella geografia (S. James, 1990; Sibley, 1991), i lavori su infanzia e spazi della quotidianità (Aitken, 1994; Sibley, 1995), la contrapposizione alle geografie del mondo degli adulti e le pratiche di resistenza, contestazione e appropriazione di cui bambini e adolescenti sono fautori (Valentine, 1996b) rappresentano i principali assi lungo i quali si sviluppa il discorso delle Children's Geographies nel corso degli anni Novanta.

Se negli anni Novanta la geografia dei bambini muove i primi passi, è negli ultimi due decenni che si struttura e si ramifica combinando un'inedita varietà di approcci e tematiche (Agnew et al., 2015, p. 270; Bartos, 2012; Belotti, 2000; Elwood e Mitchell, 2012; Holt, 2004; Moosa-Mitha, 2005; Skelton e Valentine, 2003; Valentine e Holloway, 2000; Wood, 2012)6. Nel 2003 viene inaugurata su iniziativa di Hugh Matthews Children's Geographies, una nuova rivista dedicata alle geografie dei bambini che negli anni è divenuta un imprescindibile punto di riferimento per quest'area di studi. Nel 2013 la rivista Space and Polity dedica uno special issue alle geografie politiche dei bambini (Kallio e Häkli, 2013) e nello stesso anno la conferenza annuale RGS-IBG ospita la prima plenaria sulle Children's Geographies. È all'interno di questo quadro che le discussioni sulle geografie politiche dei bambini in diversi contesti geografici si moltiplicano, intrecciando scale e spazi differenti e dando vita a un campo di studi eterogeneo e consolidato (Agnew et al., 2015, p. 218). Il perno epistemologico di questo campo è rappresentato, come vedremo nel prossimo paragrafo, dal riconoscimento del ruolo attivo della spazialità nella costruzione dell'agency dei bambini. La centralità dei luoghi nell'e-

${ }^{5}$ Se la sistematizzazione di questa nuova branca oggi unanimemente riconosciuta con il nome di "Children's Geographies" risale alla seconda metà degli anni Novanta e ai primi anni Duemila, la costruzione di un discorso sulla relazione fra i bambini e la spazialità può contare su articoli e volumi antecedenti allo spartiacque del 1989, la cui eredità teorica arriva fino a oggi (Ward, 1978; Katz, 1986; Moore, 1980, 1986).

${ }^{6}$ In Italia la riflessione sulle geografie dei bambini ha riguardato soprattutto gli aspetti legati alla didattica, con particolare riferimento alla scuola dell'infanzia e primaria (De Vecchis, 2011; Giorda, 2006, 2014; Giorda e Puttilli, 2011; Squarcina, 2009). La più completa trattazione sulle relazioni tra spazio e infanzia in geografia è rappresentata dal volume di Stefano Malatesta Geografia dei bambini (Malatesta, 2015). 


\section{Chiara Giubilaro}

sperienza politica dei bambini è infatti un tratto distintivo della geografia politica dei bambini e ne determina non soltanto i contenuti ma anche gli approcci e le metodologie. Nel quadro dell'insopprimibile asimmetria di potere che attraversa la relazione fra bambini e adulti, la spazialità dei bambini si presenta nella forma di una continua negoziazione (Kallio e Häkli, 2011, p. 105). Muovendosi all'interno di spazi pensati e costruiti da adulti, i bambini si trovano infatti a dover attivare strategie di adattamento, resistenza o appropriazione entro contesti, posizionamenti e identità scelti per loro da altri. Attraverso questo complesso sistema di negoziazioni fatto di pratiche, rappresentazioni, simboli e azioni, i bambini producono e riproducono continuamente gli spazi che attraversano e abitano nel loro quotidiano (Di Napoli, 2012; Malatesta, 2015).

Prima di addentrarci nel dibattito sull'agency dei bambini e sul ruolo che il diritto ad aspirare può avere al suo interno, due considerazioni si rendono necessarie. Come molti delle autrici e degli autori a cui farò riferimento hanno ampiamente dimostrato, l'infanzia non rappresenta in alcun modo una fase biologica data, ma al contrario essa è sempre una costruzione discorsiva, i cui confini cambiano nello spazio e nel tempo (Aries, 1962; Hendrick, 1990). Sebbene il dibattito sia aperto, in questo lavoro considereremo 'bambini' i soggetti di età compresa fra i cinque e i sedici anni, in linea con gran parte della letteratura sul tema (Matthews e Limb, 1999, p. 63) e con la scelta dei partecipanti al percorso di ricerca-azione. Seguendo una convenzione propria della geografia politica dei bambini includeremo così anche soggetti convenzionalmente considerati adolescenti, nella convinzione che quel che è più rilevante nelle pratiche spaziali di questa fascia di età sia la differenza - e, alle volte, la contrapposizione - con il mondo degli adulti (Valentine, 1996b). Naturalmente, l'infanzia non può in alcun modo essere considerata una categoria omogenea e qualunque discorso intorno alla spazialità dei bambini deve tenere conto delle differenze che attraversano i soggetti che ne fanno parte (Valentine, 1996a, p. 587). Sebbene alla categoria di infanzia siano spesso associati tratti quali la vulnerabilità, la dipendenza, l’innocenza o la spensieratezza, non bisogna dimenticare che questi sono parte di una visione stereotipata che spesso non trova alcun riscontro nella materialità delle esperienze di coloro che appartengono a questa fascia di età. La complessità e l'ambiguità costitutive dell'infanzia come costruzione sociale vanno infatti affrontate in una prospettiva intersezionale che tenga conto di altri assi identitari, quali la classe, la razza, l'etnicità, il genere e così via. Come sottolineano Matthews e Limb, esistono tante 'infanzie' quanti i bambini che le incarnano, con le loro differenze e le loro specificità (1999, p. 65). Per questa ragione non c'è discorso sull'infanzia, compresi quelli qui presentati, che possa prescindere dal riconoscimento del carattere necessariamente instabile e provvisorio di ogni sua affermazione generalizzante. 


\section{Il diritto ad aspirare nelle geografie dei bambini}

3. Reclamare il diritto ad aspirare. - In una recente raccolta di saggi dal titolo Il futuro come fatto culturale, dedicata alla sua decennale esperienza di ricerca-azione negli slum di Mumbai, l'antropologo indiano Arjun Appadurai si interroga su come ripensare la relazione fra cultura e futuro. La nozione di cultura, troppo a lungo ancorata a tradizioni e patrimoni del passato, va secondo l'autore radicalmente riorientata verso il futuro. In questo senso, coltivare aspirazioni e immaginare scenari alternativi rappresentano una capacità culturale che non può in alcun modo essere trascurata, anche per via del legame fra questa e la povertà urbana. Le aspirazioni, infatti, non sono mai semplicemente individuali, ma derivano da norme sociali e culturali più ampie: "[Questo] Significa che quanto meglio stai (in termini di potere, dignità e risorse materiali), tanto maggiore sarà probabilmente la consapevolezza dei collegamenti fra la maggiore o minore vicinanza degli oggetti a cui aspiri” (Appadurai, 2013, p. 258, trad. it. 2014). La possibilità stessa di prefigurarsi un futuro è il prodotto di vincoli normativi, condizioni sociali, risorse materiali. Negli slum di Mumbai - racconta Appadurai - questa capacità è a rischio, assottigliata da disuguaglianze e privazioni. Se la capacità di aspirare si riduce all'inasprirsi delle condizioni di povertà e vulnerabilità, allora diviene decisivo lavorare per cercare di rafforzarla attraverso la pratica, l'esplorazione e la protesta (2013, p. 260). Incrementare la capacità di immaginare futuri possibili e rivendicarli attraverso la forma della protesta sono, come vedremo, le due tattiche suggerite da Appadurai per cercare di aprire nuovi spazi di agency politica.

Sebbene la riflessione sulle aspirazioni non sia esplicitamente riportata al mondo dell'infanzia, è proprio sul terreno delle geografie dei bambini che questa può da un lato rivelare con forza il suo portato politico e la sua carica trasformativa, dall'altro contribuire a scardinare alcune ortodossie concettuali che contraddistinguono il dibattito interno alla disciplina. Come abbiamo più su mostrato, il perno epistemologico intorno al quale negli anni è stata costruita la geografia politica dei bambini è consistito nel riconoscimento dei bambini come attori sociali e politici. L'insistito richiamo all'agency dei bambini, come segnalano in una recente critica Sarah Holloway, Louise Holt e Sarah Mills (Holloway et al., 2019), è divenuto una sorta di mantra replicato con scarsa attenzione teorica. Attraverso una fitta rassegna di lavori sul tema, le autrici pongono il concetto di agency al centro di un'attenta ricognizione critica che prova a scandagliare le differenti forme e strutture attraverso cui si articola la soggettività dei bambini e a rimettere in discussione i postulati che spesso la accompagnano. Per esempio, la presupposizione che l'agency dei bambini sia sempre positiva si scontra con la materialità dei casi e dei contesti, rimuovendo dalle nostre analisi le situazioni in cui i bambini riproducono strutture di oppressione e disuguaglianza (Sparks, 2016). O, ancora, l'enfasi sull'autonomia dei bambini in quanto attori sociali indipendenti rischia di mancare il carattere profondamente relazionale della loro agency, vale a dire la 
loro capacità di agire in contesti di forte dipendenza inter- e intra-generazionale (Bartos, 2012). Come sottolinea Deborah Durham, questa celebrazione irriflessa dell'agency dei bambini poggia peraltro su una sorta di paradosso epistemologico, dal momento che si fonda su un'idea liberale di soggettività autonoma che storicamente si afferma in seno a quella stessa tradizione illuminista che esclude bambine e bambini dal proprio orizzonte teorico e politico (Durham, 2008, p. 152).

Se vogliamo raccogliere la sfida critica lanciata da queste autrici e problematizzare la questione dell'agency nelle geografie dei bambini, la categoria di aspirazione può a mio avviso rappresentare un utile strumento teorico per almeno tre ragioni. Anzitutto, il suo costitutivo legame con la dimensione del futuro consente di scartare una visione della soggettività come qualcosa di compiuto e predeterminato e, proiettandola costantemente in avanti, suggerisce quanto questa sia al contrario contingente e mutevole, come si adatti di volta in volta ai contesti e alle pratiche. Le aspirazioni, infatti, non sono necessariamente positive e il loro farsi è sempre il prodotto di incontri, circostanze e condizioni non prevedibili. In secondo luogo, come abbiamo già ricordato, l'aspirazione nella formulazione che ne offre Appadurai è sempre una capacità collettiva (Appadurai, 2013, p. 258, trad. it. 2014). Ripensare l'agency dei bambini come esito di pratiche di aspirazione significa assegnare un ruolo chiave alla dimensione relazionale: le aspirazioni non sono desideri individuali, ma al contrario si costruiscono e si coltivano dentro le relazioni. La loro fragilità e la loro forza poggiano esattamente su questo presupposto. Così come sono esposte ai colpi della povertà e delle condizioni di vulnerabilità sociale, culturale ed economica, allo stesso modo possono essere consolidate e ampliate attraverso un costante lavoro sulla capacità di rivendicare spazi di speranza e sulla necessità di farlo insieme. Infine, per le ragioni che abbiamo appena illustrato, le aspirazioni consentono di politicizzare la questione dell'agency nelle geografie dei bambini. La categoria di aspirazione, infatti, poggia su una lettura della temporalità futura fortemente politica e la rende operativa nel presente. Nel tentativo di scartare la logica che guarda al futuro come a una scena di là da venire su cui proiettare le necessità o le speranze del presente attraverso una relazione di successione, Ben Anderson suggerisce per esempio di ripensare la categoria di futuro come insieme aperto e plurale di possibilità che si dispiegano nella contingenza del qui e ora (Anderson, 2010). Una geografia del possibile, dunque, che si propone di indagare questa multiforme 'presenza del futuro' negli spazi vissuti del quotidiano e che sembra riecheggiare la lezione di Giuseppe Dematteis. Nel suo Le metafore della terra, il geografo torinese invita a tornare a pensare e a praticare la geografia nella sua forma 'fantastica', intesa come ricerca di alternative capaci di sottrarsi e di ribellarsi all'ordine esistente delle cose e alle sue necessità. "Descrivere le condizioni dell'emergere nel presente di ciò che è nuovo e inatteso" (Dematteis, 1985, p. 121) è la strada che la geografia deve seguire, avviando un serio dialogo con il 


\section{Il diritto ad aspirare nelle geografie dei bambini}

mondo in trasformazione. In questo senso, la categoria di aspirazione può tenere insieme da una parte la critica all'ordine esistente delle cose, dall'altra il potenziale immaginativo che ogni politica del cambiamento richiede.

Nei prossimi due paragrafi cercheremo di comprendere come la riflessione sulle aspirazioni urbane possa incrociare le geografie materiali dei bambini e delle bambine attraverso l'analisi del caso del quartiere CEP di Palermo. Dapprima proporrò una contestualizzazione socio-spaziale e storica del CEP, in seguito cercherò di restituire in chiave critica i processi che mi hanno vista direttamente impegnata sul territorio durante il percorso di ricerca-azione che ho qui condotto.

4. Il CEP fRa politiche di abbandono e diritto alla SopravVivenza. - A osservarlo dall'alto delle immagini satellitari, il quartiere $\mathrm{CEP}^{7}$ di Palermo si presenta come un trapezio poggiato sull'asse di viale Michelangelo, alla periferia ovest della città (Fig. 1). Su un'area di trenta ettari, fino agli anni Cinquanta occupata da campi e agrumeti, svettano oggi all'incirca novanta edifici, la maggior parte dei quali presenta quattro o più elevazioni e caratteristiche tipiche dell'edilizia residenziale pubblica della seconda metà del secolo scorso. Qui, come vedremo, a partire dalla metà degli anni Sessanta si stabiliscono perlopiù abusivamente famiglie provenienti da diverse aree del centro storico della città e dei comuni limitrofi, ciascuna con una storia e un modo di vivere lo spazio urbano peculiari, dando vita a un tessuto sociale disomogeneo e attraversato da fratture ancora oggi ben visibili.

Con i suoi cinquemila abitanti, di cui oltre un terzo interrompe gli studi dopo la licenza elementare e almeno un quinto è formalmente disoccupato ${ }^{8}$, il CEP $^{9}$ rappresenta oggi uno dei quartieri più violentemente marginalizzati di Palermo ${ }^{10}$, che alle gravi condizioni di vulnerabilità socioeconomica caratteristiche di molte altre zone della città unisce una storia di abbandono da parte non solo delle istituzioni ma anche del terzo settore che ha impresso segni profondi sul territorio

7 Dal momento che, nonostante una delibera comunale del 1997 abbia sostituito l'acronimo "CEP" (Comitato di Coordinamento di Edilizia Popolare) con "San Giovanni Apostolo" (dal nome della parrocchia), gli abitanti del quartiere continuano a utilizzare il toponimo originario, si è scelto nella ricerca di mantenere questa denominazione.

${ }^{8}$ Dati elaborati attraverso il Sistema Informativo Geografico dell'Istat (Gistat), 2011.

9 Sebbene l'UPL San Giovanni Apostolo abbia un'estensione maggiore, ho preferito restringere il campo di ricerca e di intervento entro i confini del cosiddetto trapezio per ragioni di ordine storico (il nucleo originario del quartiere è contenuto entro questi confini e diverge per tipologia edilizia e caratteristiche abitative dalle aree di più recente costruzione), sociale (l'area esterna ai confini presenta caratteristiche socio-economiche profondamente differenti), di autorappresentazione (i confini percepiti dai residenti ricalcano, con alcune ovvie variazioni, quelli del trapezio).

${ }^{10}$ Nonostante condivida con il più noto quartiere ZEN di Palermo decenni di politiche di abbandono e disinvestimento (Fava, 2008), il CEP si contraddistingue per una carenza strutturale di interventi di inclusione sociale e contrasto alle disuguaglianze. Come vedremo, gli unici presidi sociali presenti nel quartiere sono l'associazione legata alla parrocchia e la scuola. 


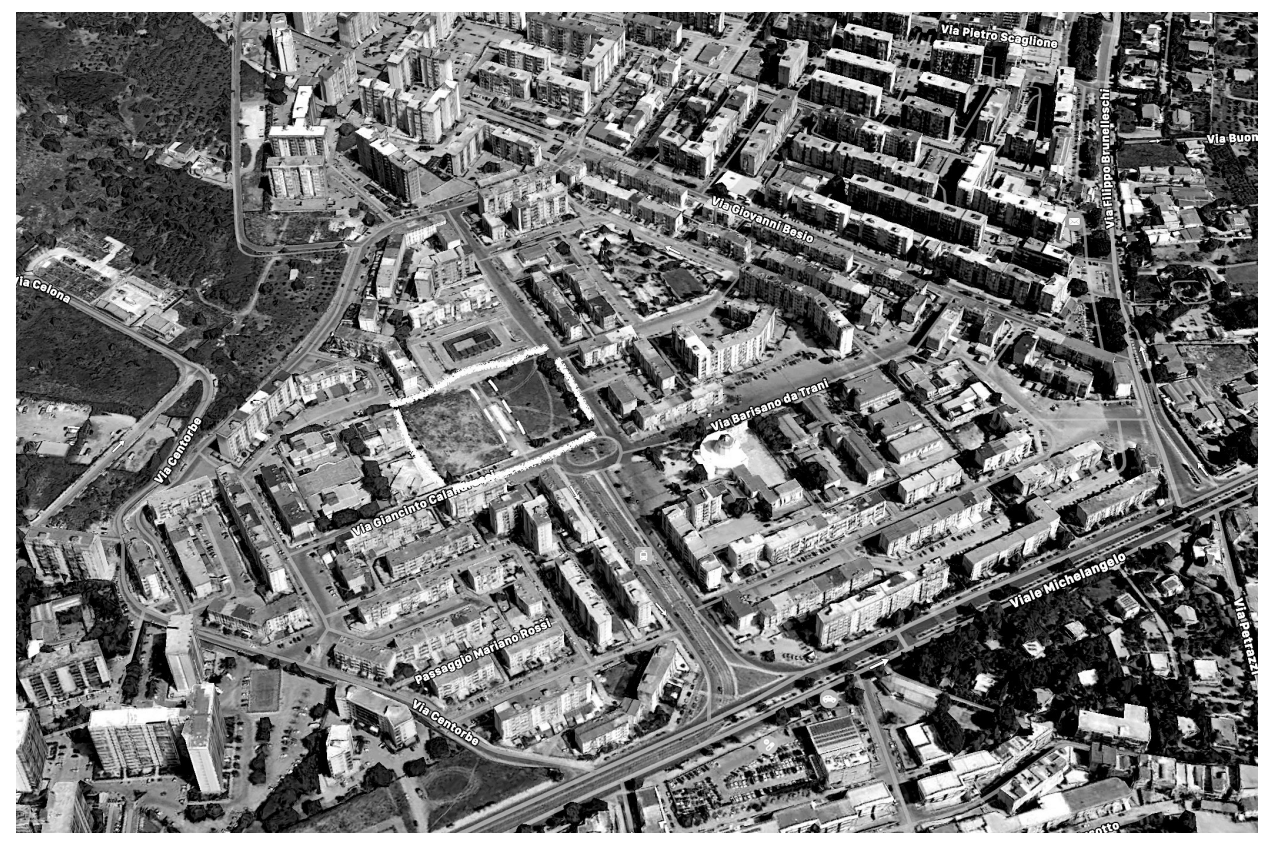

Fonte: rielaborazione dell'autrice da GoogleMaps.

Fig. 1 - Il quartiere CEP/San Giovanni Apostolo e particolare dell'area di via Calandrucci

(Giampino et al., 2020). Riprendendo le parole di Ananya Roy, quel qui mi preme qui sottolineare non è tanto la condizione di marginalità in cui versano il CEP e molte altre 'periferie' del mondo, quanto la capacità di marginalizzazione come tecnologia peculiare di governo urbano (Roy et al., 2019, p. 28). Come vedremo, infatti, le politiche di abbandono che caratterizzano quest'era di neoliberismo urbano hanno confinato spazi e persone in una dimensione apparentemente slacciata dal resto della città e dalle sue dinamiche di governo, imprimendosi violentemente sulle loro storie e geografie (Peck, 2012; Tulumello et al., 2019; van Lanen, 2020).

Oggi all'incirca un abitante ogni cinque al CEP è una bambina o un bambino che, come abbiamo visto nei precedenti paragrafi, fa esperienza del proprio quartiere e degli spazi che lo compongono secondo modalità del tutto peculiari. La forte presenza dei bambini in questa porzione di città è un tratto che ricorre nella sua storia oltre che nella sua geografia. Specie durante la prima fase insediativa, intorno agli anni Sessanta, moltissimi erano i bambini e le bambine che si stabilirono negli edifici di nuova costruzione insieme alle proprie famiglie e che costruirono pratiche e forme di appropriazione di quegli spazi ancora in costruzione. Se la geografia politica dei bambini fornisce approcci e strumenti utili a leggere qualunque 


\section{Il diritto ad aspirare nelle geografie dei bambini}

spazio in questa prospettiva, il quartiere CEP rappresenta sicuramente un terreno di indagine particolarmente fertile per questo genere di riflessioni.

La genesi del CEP è legata a doppio filo con la storia degli interventi di edilizia residenziale pubblica in Italia e, più nello specifico, con lo stretto legame fra questione abitativa e sviluppo urbanistico nella città di Palermo (Badami, 2012; Picone e Schilleci, 2012) ${ }^{11}$. Sebbene il progetto del nuovo quartiere-satellite ${ }^{12}$ con le sue aree dedicate ai servizi e alle strutture di interesse collettivo, all'edilizia pubblica e a quella di iniziativa privata rispondesse a una logica di integrazione, la sua realizzazione si rivela presto una radicale sconfessione di tali presupposti. Le lunghe e complesse fasi progettuali e realizzative da un lato e le impreviste dinamiche insediative dall'altro hanno infatti tradito le logiche che avevano sostenuto il progetto originario e aggravato i processi di marginalizzazione socio-spaziale ancora oggi in atto (Giampino et al., 2020, pp. 45-6). In particolare, il terremoto del 1968 trasforma radicalmente gli assetti urbani e sociali del CEP e dell'intera città, portando nelle settimane successive oltre cinquemila sfollati a trasferirsi dal centro storico nel nuovo quartiere. Con il loro arrivo, la composizione sociale del CEP cambia radicalmente. I 'nuovi abitanti', nelle testimonianze ${ }^{13}$ di chi si era trasferito negli anni precedenti, introdurrebbero degrado e delinquenza fra le vie del quartiere, dando avvio a una lunga storia di fratture e conflitti interni le cui risonanze arrivano ai nostri giorni. A partire dagli anni Settanta, tuttavia, la mancanza di servizi, le politiche di abbandono e il controllo mafioso del territorio trovano un argine nel ruolo attivo della parrocchia e nella costituzione di un comitato di quartiere, un organo informale composto da abitanti, politici, volontari. Nonostante gli sforzi, analfabetismo, disoccupazione, precarietà abitativa, sposi-bambini ${ }^{14}$, violenza sulle donne ${ }^{15}$ e sui minori rappresentano il prodotto tangibile della mancanza di qualunque investimento sulle politiche sociali nel quartiere. La scuola, la parrocchia e l'associazione costituiscono infatti nell'arco di questa fase gli unici tre soggetti in grado di fare da argine al vuoto delle istituzioni e di costruire spazi di aggregazione in un quartiere ancora attraversato da violente fratture fra vecchi e nuovi abitanti. Le 'isole' di divisione e diffidenza di cui scriveva il parroco del quartiere nei primi anni Settanta (Russo, 2009) sembrano imprimersi sulle geografie del CEP attraverso tutte le fasi della sua storia.

${ }^{11}$ Le principali fonti di cui mi sono servita in quest'opera di ricostruzione sono (I) le interviste e le storie di vita raccolte con gli abitanti, specie con coloro che hanno vissuto da bambini la genesi del quartiere e i suoi primi sviluppi; (II) lo spoglio di alcuni quotidiani locali e nazionali, con particolare riferimento agli eventi successivi al terremoto del 1968; (III) le poche fonti bibliografiche riguardanti il CEP e la sua storia.

${ }^{12}$ Il progetto rientra fra gli interventi del secondo settennio del piano INA-casa realizzati fra il 1957 e il 1964 (Di Biagi, 2002).

${ }^{13}$ Interviste in profondità svolte fra aprile e maggio 2019.

${ }^{14} \mathrm{Si}$ rimanda all'articolo di Ugo Baduel, "Nel rione degli sposi bambini”, in L'Unità (17.04.1976).

${ }^{15}$ Intorno alla sezione del PCI e all'attivista Lucia Mezzasalma gravitava un buon numero di donne del quartiere che aveva aderito all'UDI e promuoveva battaglie di emancipazione femminile. Cfr. Baduel, "Parlano le donne di Palermo", in L'Unità (9.04.1976). 


\section{Chiara Giubilaro}

5. Uno SPAZio per aspirare: fare RiCERCA-AZIONe al CEP. - La prima volta che sono arrivata al CEP la mia attenzione è stata catturata da un enorme spazio aperto, appena oltre la fermata del tram, la cui vista era incorniciata dal profilo di uno dei rilievi che circondano la città di Palermo: tre aree contigue di poco più di un ettaro, due delle quali in evidente stato di abbandono, la terza che invece mostrava i segni di diversi tentativi di recupero, nessuno dei quali poteva dirsi completamente riuscito (Fig. 2). Nel corso delle settimane successive, l'immagine di quel vuoto si è sovrapposta da una parte alle linee e alle forme dei piani che sul finire degli anni Cinquanta avevano previsto in questo spazio la realizzazione di un numero sorprendente di servizi per il quartiere, dall'altra alle aspirazioni che molti prima di me avevano proiettato su quello spazio, riuscendo in alcuni casi a realizzarle per brevi periodi di tempo e progetti estemporanei. L'area di via Calandrucci aveva ospitato un campo da bocce, due campi da calcio, spogliatoi, aree ristoro e giostre per i più piccoli. Nulla di tutto questo aveva impresso segni permanenti sul territorio, ma quando ho cominciato il laboratorio di quartiere con le ragazze e i ragazzi dell'Associazione San Giovanni Apostolo ho capito che segni ben più durevoli erano rimasti nell'immaginario dei miei interlocutori.

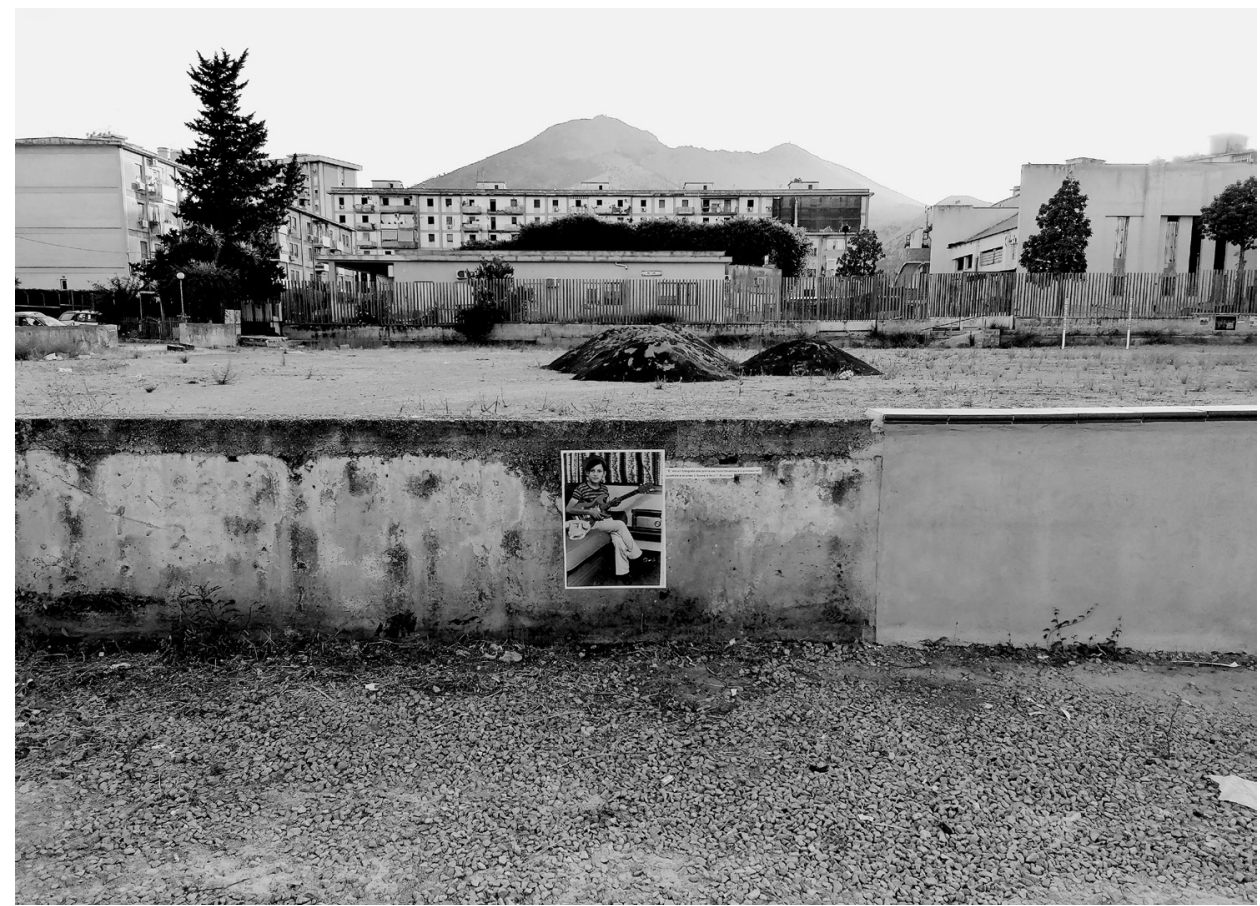

Fonte: fotografia dell'autrice.

Fig. 2 - L'area di via Calandrucci 


\section{Il diritto ad aspirare nelle geografie dei bambini}

5.1 Metodologie differenziate. - Prima di analizzare l'esperienza laboratoriale, sono necessarie alcune considerazioni sulle metodologie adottate. Per le ragioni esplicitate nel paragrafo teorico, il presupposto epistemologico da cui questo percorso ha preso le mosse è consistito nel riconoscere che ciascun bambino costruisce e organizza uno specifico sapere sugli spazi di cui fa esperienza, nella formazione del quale interferiscono le differenze di classe, di genere e così via. Spostare l'attenzione dalla dimensione della percezione, frequentemente richiamata negli studi sulla geografia dei bambini (Ansell, 2009; Mitchell et al., 2007) a quella del sapere significa riconoscere ai bambini il ruolo di soggetti attivi anche nella produzione e nella condivisione di veri e propri saperi geografici (Delcasino et al., 1997, p. 40). Seguendo i presupposti della ricerca-azione partecipativa (Kindon et al., 2007; Lambert-Pennington e Saija, 2020), la sfida è allora consistita nel riconoscere da una parte l'esistenza di una pluralità di saperi, dall'altra l'impossibilità di ordinarli gerarchicamente. Combinare insieme saperi eterogenei e alle volte contrastanti, riflettere collettivamente sui contenuti di tali saperi e sulle modalità attraverso cui condividerli, ha richiesto una mobilitazione di metodologie e approcci differenziati, nel difficile tentativo di costruire un 'campo in comune' (Katz, 1994, p. 70). Per queste ragioni ho scelto di utilizzare una metodologia mista qualitativa (Meth e Mcclymont, 2009), modulando di volta in volta tecniche e approcci a seconda dei contesti, dei soggetti, delle interazioni. La contingenza e la peculiarità delle relazioni che hanno prodotto il campo della ricerca hanno pertanto richiesto una mobilitazione di strumenti eterogenei, ciascuno calibrato sul differenziale di potere fra il mio posizionamento e quello degli attori coinvolti. Così, se nell'interazione con gli attori istituzionali (assessori, dirigenti, consiglieri di circoscrizione) ho fatto prevalentemente ricorso alle metodologie tradizionali della ricerca qualitativa, in primis interviste in profondità e analisi critica del discorso (Wodak e Meyer, 2009), maggiori spazi di sperimentazione sono stati invece ricercati nelle relazioni con i residenti, con i quali la principale tecnica utilizzata è stata quella delle storie di vita condotta entro spazi di interazione il più possibile informali (Rogaly, $2015)^{16}$. Nel corso del laboratorio con le ragazze e i ragazzi dell'Associazione San Giovanni Apostolo, infine, le metodologie tradizionali (questionari, sopralluoghi, tecniche visuali) sono state oggetto di una rivisitazione non sempre riuscita in chiave partecipativa. Ciascuna di esse, infatti, è stata illustrata ai ragazzi attraverso delle simulazioni e poi rielaborata da loro in modo che potessero utilizzarla in prima persona per esplorare le geografie del CEP. Così, i ragazzi sono stati chiamati a costruire e somministrare questionari, pensare e organizzare sopralluoghi, praticare

${ }^{16}$ Durante il percorso di ricerca sono state raccolte ventiquattro interviste in profondità e sei storie di vita dei residenti nel quartiere. In uno di questi casi, durante l'incontro, gli album fotografici di famiglia sono stati oggetto di photo-elicitation (Bignante, 2010), trasformando gli stimoli visuali nell'origine di racconti legati al quartiere. 
la tecnica del photovoice (Breny e McMorrow, 2021; Frisina, 2012; Johnsen et al., 2008; Lombardi-Diop e Romeo, 2005) rideclinata in versione social. Questo assetto metodologico il più possibile destrutturato è stato un tentativo di risposta alla necessità di costruire spazi, strumenti e condizioni in forza dei quali i soggetti con cui ho interagito potessero prendere la parola e dare loro stessi voce e occhi alle proprie aspirazioni.

5.2 Il laboratorio di quartiere. - Il laboratorio di quartiere e le relazioni che lo hanno attraversato sono stati la mia prima chiave d'accesso al CEP e alle sue dinamiche. Il lavoro teorico e pratico sugli orizzonti di aspirazione dei ragazzi rispetto allo spazio urbano e alle sue possibili trasformazioni ha rappresentato il terreno sul quale ho provato a costruire temi, strumenti e attività del laboratorio (Fig. 3). Iniziato ad aprile del 2019 e conclusosi a giugno dello stesso anno, il laboratorio ha coinvolto un gruppo di ragazze e ragazzi di età compresa fra gli undici e i quattordici anni ${ }^{17}$ e si è strutturato attraverso incontri settimanali nei locali dell'Associazione e per le strade del quartiere. Sopralluoghi tematici, tecniche di autofotografia, mappature dal basso, dibattiti e dialoghi hanno fatto da sfondo alla costruzione del gruppo e al mio graduale e non sempre riuscito inserimento al suo interno. Sebbene molte siano le questioni connesse alla costruzione del laboratorio, l'attenzione si concentrerà qui sulle pratiche spaziali attraverso cui il percorso di ricerca-azione si è dispiegato e su come le dinamiche di partecipazione abbiano contributo a produrre spazi di relazione, dialogo e conflitto all'interno dei quali ogni posizionamento è divenuto irrimediabilmente problematico. Nella parte finale del paragrafo ci soffermeremo infine su alcune delle attività svolte nel corso del laboratorio.

La città ha rappresentato nel corso del laboratorio una scala ambigua con cui interagire. La percezione di una distanza difficile da colmare ha accompagnato i discorsi e le rappresentazioni dei partecipanti. Il tram, che dal 2015 collega in una dozzina di minuti il CEP a uno snodo di trasporti vicino al centro, ha certamente accorciato questa distanza, permettendo ai ragazzi di "scendere in città" il sabato pomeriggio e trascorrere lì il proprio tempo libero. Tuttavia, il desiderio di mobilità è emerso in forme inaspettate durante uno degli incontri, quando il satellite di GoogleMaps è diventato un surrogato virtuale di viaggi e spostamenti e quella che nelle mie intenzioni avrebbe dovuto essere un'occasione per riflettere sul loro quartiere si è presto trasformata nella richiesta di sorvolare, con lo sguardo fisso sullo schermo, destinazioni "lontane", come lo stadio o la spiaggia.

${ }^{17}$ Fin dal primo incontro mi è stato affiancato un mediatore scelto fra i giovani volontari del centro aggregativo. 


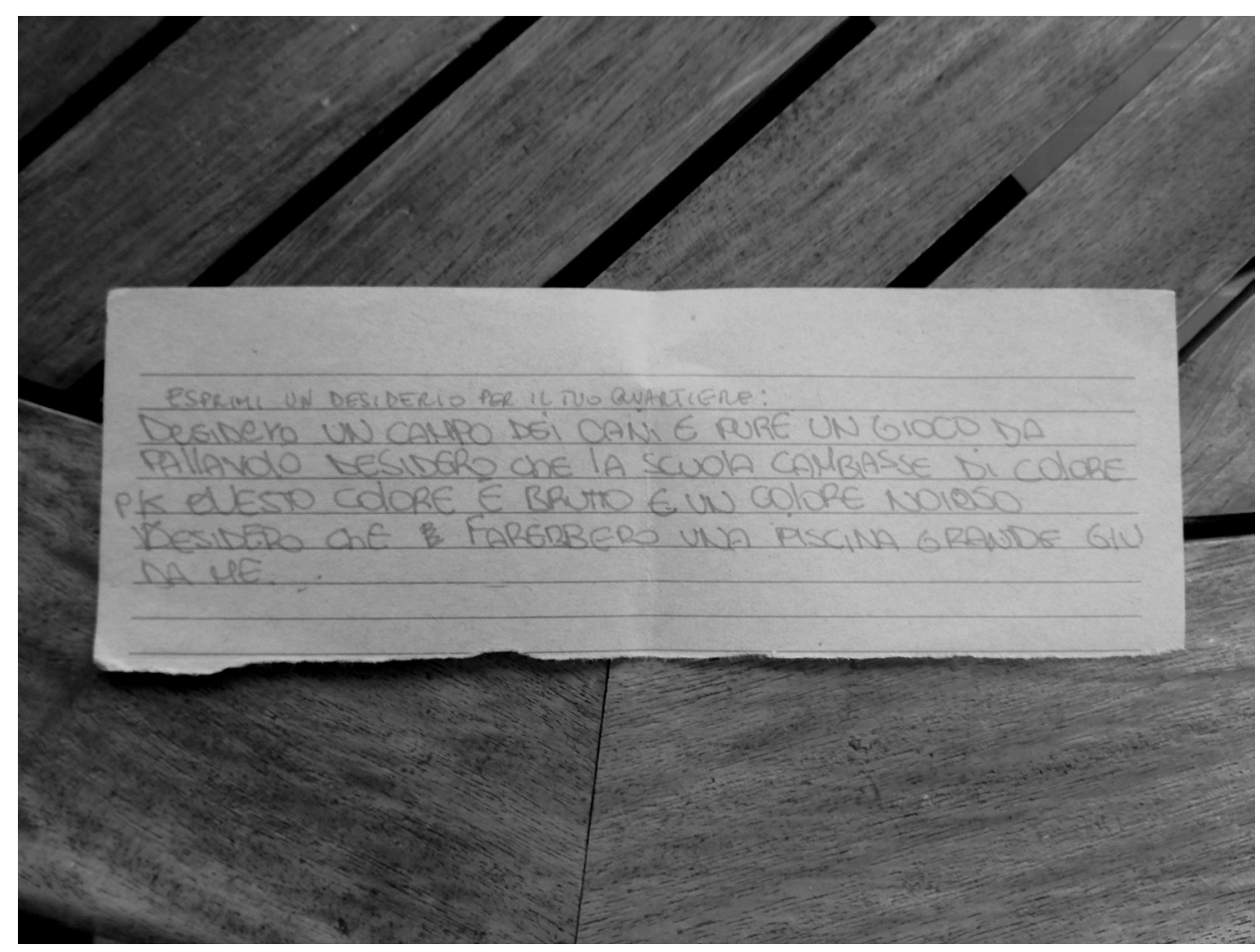

Fonte: fotografia dell'autrice.

Fig. 3 - "Esprimi un desiderio per il tuo quartiere"

Se costruire un percorso di ricerca azione partecipativa significa inevitabilmente contribuire a produrre spazi di relazione e di conflitto, fra questi un ruolo di primo piano ha avuto l'aula dove per alcune settimane ho incontrato le ragazze e i ragazzi e le trasformazioni che l'hanno attraversata nel corso delle settimane: la mia posizione al suo interno e, forse più ancora, le loro si sono ri-assemblate innumerevoli volte e il dialogo fra noi ha lasciato gradualmente spazio all'emergere di pratiche e modi di interazione più fluidi. Questo non ha naturalmente annullato il differenziale di potere presente all'interno dell'aula, con il quale ho dovuto fare faticosamente i conti in più occasioni, ma mi ha permesso di riflettere su come rimetterlo in circolo attraverso pratiche e relazioni. L'atteggiamento dei ragazzi nei primi giorni mi aveva per esempio rivelato quanto poco fossero abituati a forme meno gerarchiche di interazione e quanto invece si trovassero a proprio agio dentro ruoli perlopiù passivi e assetti rigidamente codificati. Non è casuale che questi assetti venissero stravolti in occasione dei sopralluoghi di quartiere: lasciare l'aula e percorrere i larghi viali del CEP significava sperimentare inedite configurazioni di 
relazione, dove non soltanto i ragazzi riuscivano a rinegoziare quella forma di thin agency tipica dei contesti altamente restrittivi (Klocher, 2007; Payne, 2012) che in aula risultava in qualche modo inibita, ma anche i più restii a mettersi in gioco trovavano nello spazio di quartiere un'occasione per raccontare e raccontarsi.

Le rappresentazioni, specie quelle visuali, sono l'altro campo su cui le possibilità di agency dei partecipanti si sono manifestate con maggiore forza. Mappare il quartiere, fotografarne i punti di forza e quelli di debolezza, inventare hashtag e storie per raccontarlo attraverso Instagram sono state attività decisive nella costruzione del percorso. Fin dalle prime interazioni con e fra i partecipanti è emerso quanto la questione della rappresentazione del quartiere fosse sempre il prodotto di una tensione fra auto- ed etero-rappresentazione e quanto profondamente lo sguardo sul quartiere interferisse con qualunque tentativo di raccontare e raccontarsi. Ne è un esempio quanto accaduto durante il primo incontro del laboratorio, quando nel discutere dei nomi del quartiere e del loro significato (cfr. nota 7) un ragazzo ha sciolto l'acronimo CEP in 'Centro Elementi Pericolosi', rivendicando l'efficacia e la forza di una definizione connessa alla visione stigmatizzante che contraddistingue la relazione fra il quartiere e il resto della città e innescando così un'ampia gamma di reazioni e commenti fra i partecipanti.

Le metodologie visuali, come già accennato, hanno avuto un ruolo chiave nel processo di costruzione del laboratorio, in linea con quanto espresso dagli stessi partecipanti in occasione del brainstorming preliminare in cui è stato domandato loro quali fossero le loro aspirazioni per il laboratorio che di lì a poco avremmo svolto. Due incontri sono stati così dedicati all'organizzazione del photo-walk (Fig. 4), una camminata di quartiere realizzata utilizzando la tecnica del photovoice (Pyyry, 2013; Templeton, 2020). I partecipanti sono stati dapprima suddivisi in due gruppi, ciascuno dei quali è stato chiamato a immaginare e costruire un itinerario che toccasse i luoghi più significativi della loro quotidianità, con particolare attenzione al tema delle aspirazioni urbane. Per ciascuna tappa avrebbero dovuto indicare una tipologia fra le seguenti: luoghi in cui mi sento a mio agio; luoghi in cui non mi sento a mio agio (efficacemente definiti dai partecipanti i luoghi dello scafazzo); luoghi che mi piacerebbe poter trasformare. Il kit fornito ai ragazzi la mattina della camminata constava soltanto di una carta del quartiere e una penna colorata per tracciare gli itinerari e segnare alcune annotazioni. Ciascuno di loro aveva inoltre con sé il proprio smartphone per raccontare il quartiere attraverso la fotografia. Abbiamo così creato un profilo Instagram dedicato al laboratorio dove condividere le fotografie nella forma di post o di storie, utilizzando una serie di hashtag precedentemente discussi insieme ${ }^{18}$.

${ }^{18}$ Il profilo è stato successivamente rimosso per via di una sovrapposizione con il profilo dell'Associazione San Giovanni Apostolo e della confusione che avrebbe potuto generare. 
Nel corso del photo-walk, i ragazzi mi hanno guidata lungo i vicoli, le strade e le piazze del CEP, raccontandomi storie legate a persone e attività che non avevo mai sentito nel corso dei tradizionali sopralluoghi svolti nei mesi precedenti all'avvio del laboratorio. Ho potuto apprendere dell'esistenza di una sorta di microeconomia parallela, che ricalibra i costi di gelati e bibite per i bambini e gli adolescenti del quartiere. Grazie ai loro racconti ho visto interdetti e sbarramenti difficilmente leggibili con i tradizionali strumenti della ricerca urbana, come per esempio le marcate differenze di genere nell'uso di alcuni spazi pubblici, a conferma del carattere intersezionale a cui devono costantemente essere riportati i nostri discorsi sull'infanzia (Matthews e Limb, 1999). È il caso della villa di piazza Benvenuto Cellini, spazio verde riqualificato grazie all'interessamento di uno dei politici locali e sostanzialmente interdetto alle ragazze del quartiere, che lo descrivono come un luogo di sporcizia o pericolo.

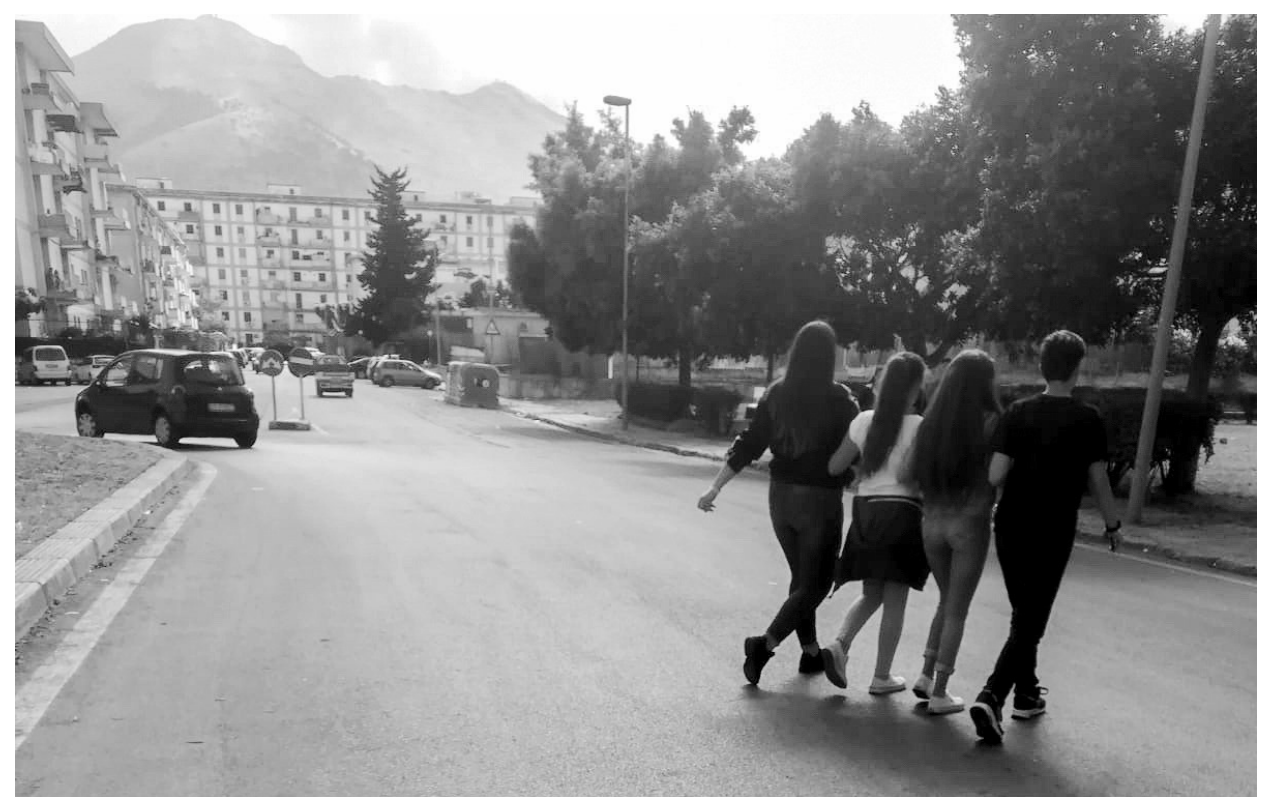

Fonte: fotografia dell'autrice.

Fig. 4 - Un gruppo di partecipanti al laboratorio durante il photo-walk

Attraverso i percorsi, i racconti e le immagini costruiti dai partecipanti un'altra geografia si è gradualmente sovrapposta a quella degli adulti, fatta di piccole botteghe, feste di piazza, memorie di campi sportivi e giostre oggi inesistenti. Le loro 
spatial stories hanno così messo in discussione le conoscenze che credevo di aver acquisito sul quartiere durante la prima fase di ricognizione attraverso gli strumenti più classici dell'inchiesta urbana. Questa geografia è stata oggetto di un processo di mappatura collettiva che ha visto le ragazze e i ragazzi impegnati nel tracciare luoghi affettivi e percorsi consuetudinari, costruire una legenda e associare a determinati simboli la propria percezione degli spazi del loro quotidiano: luoghi in disuso, luoghi da evitare, luoghi di aggregazione e luoghi da riqualificare sono confluiti sulla carta del CEP attraverso l'uso di adesivi e pennarelli colorati, proiettando così sul piano un sapere territoriale collettivo e posizionato.

È a partire da questa operazione di mappatura che si è deciso di focalizzare l'ultima fase del laboratorio sull'area di via Calandrucci, che da 'zona di disimmaginazione' è stata trasformata in un terreno su cui coltivare aspirazioni, immaginare progetti, reclamare alternative. È qui che il lavoro sulle aspirazioni urbane ha trovato un perno decisivo. L'incontro conclusivo del laboratorio ha avuto infatti per oggetto e obiettivo la costruzione di un progetto di riqualificazione dal basso del campo da gioco di via Calandrucci ${ }^{19}$. In questa occasione, per la prima volta, il lavoro portato avanti nelle settimane precedenti sul diritto ad aspirare a spazi migliori per il proprio quartiere mi è sembrato avesse, almeno in parte, innescato alcuni cambiamenti nel modo in cui i ragazzi percepivano e vivevano le proprie controverse geografie. Il campo di via Calandrucci è diventato lo spazio su cui proiettare aspirazioni collettive: un campo da calcio, un campo da pallavolo, le tribune, un palco rialzato e un'area ristoro. Questo esercizio di aspirazione, perché non alimenti processi di disillusione già fortemente radicati nel quartiere, deve essere sostenuto attraverso progettualità, azioni e politiche di lungo raggio. Solo così la capacità di immaginare spazi più vivibili e di costruire alternative all'esistente potrà rafforzarsi e attivare processi di rivendicazione e cambiamento.

6. Conclusioni. - C'è una domanda che ha accompagnato nel corso della ricerca tanto i percorsi di riflessione quanto quelli, più instabili, dell'azione e può essere così formulata: possono le aspirazioni urbane essere pensate nei termini di

${ }^{19}$ I progetti e le aspirazioni costruiti nel corso del laboratorio hanno trovato una prosecuzione nei processi di riattivazione del campo di bocce di via Calandrucci realizzati nell'ambito del progetto "Riconnessioni: percorsi di riattivazione della memoria urbana per riportare la periferia al centro" finanziato dal MIBAC per il programma "Creative Living Lab" e promosso dall'Associazione Sguardi Urbani (giugno 2019-settembre 2020), all'interno del quale ho svolto il ruolo di mediatrice territoriale. Fra le attività condotte nel quartiere allo scopo di riattivare il campo da bocce e l'area limitrofa è stato realizzato un workshop di fotografia a cura dei fotografi Andrea\&Magda, nel corso del quale gli abitanti sono stati invitati a condividere foto e memorie private e a trasformarle in una storia collettiva. Esito di questo percorso è stata la realizzazione di un album di quartiere, donato alla biblioteca dell'Associazione San Giovanni Apostolo. Una copia digitale è consultabile sul sito di Sguardi Urbani all'indirizzo: https://sguardiurbani.com/riconnessioni. 


\section{Il diritto ad aspirare nelle geografie dei bambini}

un diritto che le bambine e i bambini sono chiamati a rivendicare collettivamente per i propri quartieri? Sin dalla convenzione del 1989 sia la tutela dei minori sia la questione urbana sono ruotate intorno alla questione dei diritti. In forza del suo carattere politico e collettivo, mi sembra che il diritto ad aspirare possa superare tanto l'universalismo quanto l'individualismo caratteristici delle concezioni liberali del diritto (Moosa-Mitha, 2005), trovando nell'interdipendenza e nella relazione il proprio orizzonte di senso. In queste pagine ho cercato di mostrare come il lavoro sulla capacità di coltivare aspirazioni può in alcuni casi costituire un terreno a partire dal quale provare ad aprire spazi di azione politica e innescare processi di trasformazione urbana. Per scardinare le ortodossie teoriche della geografia politica dei bambini e rimettere in questione l'idea di agency che le sostiene (Holloway et al., 2019) è infatti necessario un cambio di passo capace di muovere dalle concrete performance che bambine e bambini mettono quotidianamente in atto e a partire da queste riflettere su come le strutture di agency si costruiscano e si manifestino, specie in contesti in di marginalizzazione, esclusione, disuguaglianze. Il percorso di ricerca-azione nel quartiere CEP di Palermo, se da un lato ha confermato il ruolo in qualche modo fondativo delle aspirazioni nel rivendicare spazi di trasformazione, dall'altro ha messo in evidenza quanto sia complesso rafforzare queste aspirazioni in chi da anni è confinato in contesti urbani caratterizzati da condizioni strutturali di abbandono e immobilismo. Per cercare di organizzare politicamente la speranza non è infatti sufficiente riempire gli spazi e i tempi dell'attesa di idee, azioni e progettualità. Occorre piuttosto mettere in atto politiche capaci di invertire i processi di disinvestimento verso questi territori e rafforzare così gli orizzonti di aspirazione di chi li abita. Affermare il diritto ad aspirare significa allora provare a reimmaginare collettivamente lo spazio e collettivamente agire perché futuri alternativi divengano presenti possibili. È questo uno dei compiti che la geografia fantastica è chiamata con ogni suo strumento a perseguire.

\section{Bibliografia}

Agnew J., Mamadouh V., Secor A.J., Sharp J., a cura di (2015). The Wiley Blackwell companion to political geography. London: Wiley Blackwell.

Aitken S.C. (1994). Putting children in their place. Washington D.C.: Association of American Geographers.

Anderson B. (2011). Preemption, precaution, preparedness: Anticipatory action and future geographies. Progress in Human Geography, 34(6): 777-98. DOI: 10.1177/ 0309132510362600

Ansell N. (2009). Childhood and the politics of scale: Descaling children's geographies? Progress in Human Geography, 33(2): 190-209. DOI: 10.1177/0309132508090980

Appadurai A. (2013). The Future as a Cultural Fact. London: Verso Books (trad. it. Il futuro come fatto culturale. Milano: Raffaello Cortina). 


\section{Chiara Giubilaro}

Aries P. (1962). Centuries of childhood: a social history of family life. New York: Random House.

Badami A. (2012). I quartieri di Edilizia Residenziale Pubblica a Palermo. In: Picone M., Schilleci F., a cura di, Quartiere e Identità. Per una rilettura del decentramento a Palermo. Firenze: Alinea.

Baraldi C., a cura di (2001). I diritti dei bambini e degli adolescenti. Una ricerca sui progetti legati alla legge 285. Roma: Donzelli.

Id., Maggioni G., a cura di (2000). Una città con i bambini. Progetti ed esperienze del Laboratorio di Fano. Roma: Donzelli.

Bartos A.E. (2012). Children caring for their worlds: The politics of care and childhood. Political Geography, 31(3): 157-166. DOI: 10.1016/j.polgeo.2011.12.003

Belotti V. (2000). La vita quotidiana di ragazze e ragazzi tra agency e ordine generazionale. In: Belotti V., a cura di, Costruire senso, negoziare spazi. Ragazzi e ragazze nella vita quotidiana. Firenze: Istituto degli Innocenti.

Bignante E. (2010). The use of photo-elicitation in field research. EchoGéo, 11: 1-20. DOI: 10.4000/echogeo.11622

Breny J.M., McMorrow S.L. (2021). Photovoice for social justice: Visual representaton in action. London: SAGE Publications.

Cancellieri A., Scandurra G., a cura di, Tracce urbane. Alla ricerca della città. Milano: FrancoAngeli.

De Vecchis G. (2011). Didattica della geografia, teoria e prassi. Torino: UTET.

Delcasino V., Dorn M.L., Gallaher C. (1997). Interview: Cindi Katz. Creating Safe Space and the Materiality of the Margins. DisClosure: A Journal of Social Theory, 6: 37-55. DOI: 10.13023/DISCLOSURE.06.03

Dematteis G. (1985). Le metafore della terra: la geografia umana tra mito e scienza. Milano: Feltrinelli.

Di Biagi P. (2002). La grande ricostruzione: il piano INA-Casa e l'Italia degli anni Cinquanta. Roma: Donzelli.

Di Napoli M. (2012). La geografia culturale e sociale dei giochi. Milano: Unicopli.

Durham D. (2008). Apathy and agency: The romance of agency and youth in Botswana. In: Cole J., Durham D., a cura di, Figuring the future: Globalization and the temporalities of children and youth. Santa Fe: SAR Press.

Elwood S., Mitchell K. (2012). Mapping children's politics: Spatial stories, dialogic relations and political formation. Geografiska Annaler, Series B: Human Geography. 94(1): 1-15. DOI: 10.1111/j.1468-0467.2012.00392.x

Fava F. (2008). Lo Zen di Palermo. Antropologia dell'esclusione. Milano: FrancoAngeli.

Frisina A. (2012). Rappresentare, autorappresentarsi. Il photovoice e gli sguardi dei Giovani musulmani d'Italia sulle loro città. In: Cancellieri A., Scandurra G., a cura di, Tracce urbane. Alla ricerca della città. Milano: FrancoAngeli.

Giampino A., Giubilaro C., Picone M. (2020). Esplorare la povertà urbana in una prospettiva mediterranea: il caso del quartiere CEP a Palermo. Archivio di Studi Urbani e Regionali. 128: 38-63. DOI: 10.3280/asur2020-128004

Giorda C. (2006). La geografia nella scuola primaria. Contenuti, strumenti, didattica. Roma: Carocci. 


\section{Il diritto ad aspirare nelle geografie dei bambini}

Id. (2014). Il mio spazio nel mondo. Geografia per la scuola dell'infanzia e primaria. Roma: Carocci.

Id., Puttilli M., a cura di (2011). Educare il territorio, educare al territorio. Roma: Carocci. Hendrick H. (1990). Constructing and reconstructions of British childhood: An interpretive survey, 1800 to present. In: James A., Prout A., a cura di, Constructing and Reconstructing Childhood: Contemporary Issues in the Sociological Study of Children. London e New York: Routledge.

Holloway S.L., Holt L., Mills S. (2019). Questions of agency: Capacity, subjectivity, spatiality and temporality. Progress in Human Geography, 43(3): 458-477. DOI: $10.1177 / 0309132518757654$

Holt L. (2004). The "voices" of children: De-centring empowering research relations. Children's Geographies, 2(1): 13-27. DOI: 10.1080/1473328032000168732

James A., Jenks C., Prout A. (1998). Theorizing childhood. New York: Teachers College Press.

Ead., Prout A. (1990). Constructing and reconstructing childhood. London e New York: Routledge.

James S. (1990). Is there a "place" for children in geography? Area, 22(3): 278-283.

Johnsen S., May J., Cloke P. (2008). Imag(in)ing 'homeless places': using autophotography to (re)examine the geographies of homelessness. Area, 40(2): 194-207. DOI: $10.1111 /$ j.1475-4762.2008.00801.x

Kallio K.P., Häkli J. (2011). Tracing children's politics. Political Geography, 30(2): 99-109. DOI: $10.1016 /$ j.polgeo.2011.01.006

Ead., Id. (2013). Children and Young People's Politics in Everyday Life. Space and Polity. 17(1): 1-16. DOI: 10.1080/13562576.2013.780710

Katz C. (1986). Children and the environment: Work, play and learning in rural Sudan. Children's Environments Quarterly, 3(4): 43-51.

Ead. (1994). Playing the Field: Questions of Fieldwork in Geography. Professional Geographer, 46(1): 67-72. DOI: 10.1111/j.0033-0124.1994.00067.x

Ead. (2001). On the Grounds of Globalization. A topography for feminist political engagement. Signs, 26(4): 1213-1234. DOI: 10.1086/495653

Kindon S., Pain R., Kesby M., a cura di (2007). Participatory action research approaches and methods: connecting people, participation and place. London e New York: Routledge.

Klocher N. (2007). An example of 'thin' agency: Child domestic workers in Tanzania. In: Panelli R., Punch S., Robson E., a cura di, Global Perspectives on Rural Childhood and Youth: Young Rural Lives. London e New York: Routledge.

Lambert-Pennington K., Saija L. (2020). To do and know something together: overcoming the obstacles and challenges. Tracce Urbane, 8: 6-18. DOI: 10.13133/ 2532-6562

Lombardi-Diop C., Romeo C., a cura di (2005). Postcolonial Italy. Challenging national homogeneity. London: Palgrave Macmillan.

Malatesta S. (2015). Geografia dei bambini. Luoghi, pratiche e rappresentazioni. Milano: Guerini Scientifica.

Masini V. (1985). Palermo: Quartieri e servizi. Palermo: Centro Studi e Iniziative "Una città per l'uomo". 


\section{Chiara Giubilaro}

Matthews H., Limb, M. (1999). Defining an agenda for the geography of children. Progress in Human Geography, 23(1): 61-90. DOI: 10.1191/030913299670961492

Meth P., Mcclymont K. (2009). Researching men: The politics and possibilities of a qualitative mixed-methods approach. Social and Cultural Geography, 10(8): 809-925. DOI: $10.1080 / 14649360903305775$

Mitchell H., Kearns R., Collins D.C.A. (2007). Nuances of neighbourhood: Children's perceptions of the space between home and school in Auckland, New Zealand. Geoforum, 38(4): 614-627. DOI: 10.1016/j.geoforum.2006.11.012

Moore R. (1980). Generating relevant urban childhood places: Learning from the 'yard'. In: Wilkinson P.F., a cura di, Innovation in play environments. London: Croom Helm.

Ead. (1986). Childhood's domain: Play and place in child development. London: Croom Helm.

Moosa-Mitha M. (2005). A difference-centred alternative to theorization of children's citizenship rights. Citizenship Studies. 9(4): 369-388. DOI: 10.1080/ 13621020500211354

Payne R. (2012). 'Extraordinary survivors' or 'ordinary lives'? Embracing 'everyday agency' in social interventions with child-headed households in Zambia. Children's Geographies, 10(3): 399-411. DOI: 10.1080/14733285.2012.726071

Peck J. (2012). Austerity urbanism. American cities under extreme economy. City, 16(6): 626-655. DOI: 10.1080/13604813.2012.734071

Picone M., Schilleci F., a cura di (2012). QUID Quartiere e identità. Per una rilettura del decentramento a Palermo. Palermo: Città e Territorio.

Pyyry N. (2013). Sensing with' photography and 'thinking with' photographs in research into teenage girls' hanging out. Children's Geographies, 13(2): 149-163. DOI: $10.1080 / 14733285.2013 .828453$

Rogaly B. (2015). Disrupting migration stories: reading life histories through the lens of mobility and fixity. Environment and Planning D: Society and Space, 33(3): 528-544. DOI: $10.1068 / \mathrm{d} 13171 \mathrm{p}$

Roy A., Tulumello S., Pozzi G. (2019). Global margins. From the production of marginalization to space of hope. Tracce Urbane, 5: 26-57. DOI: 10.13133/2532-6562

Russo G. (2009). Il quartiere CEP di Palermo. "Amore spezzato". Agrigento: Amici di S. Alfonso.

Saporiti A., Sgritta G.B. (1990). Childhood as a Social Phenomenon. National Report. Italy.

Sibley D. (1991). Children's geographies: Some problems of representation. Area, 23(3): 269-270.

Id. (1995). Families and domestic routines: Constructing the boundaries of childhood. In: Pile S., Thrift N., a cura di, Mapping the subject: Geographies of cultural transformation. London: Routledge.

Skelton T., Valentine G. (2003). Political participation, political action and political identities: Young D/deaf people's perspectives. Space and Polity, 7(2): 117-134. DOI: $10.1080 / 1356257032000133892$

Sparks H. (2016). Exploring the geographies of privileged childhoods. Geography Compass, 10: 253-267. DOI: $10.1111 /$ gec 3.12267 


\section{Il diritto ad aspirare nelle geografie dei bambini}

Squarcina E., a cura di (2009). Didattica critica della geografia: libri di testo, mappe, discorso geopolitico. Milano: Unicopli.

Staeheli L.A., Kofman E., Peake L.J., a cura di (2013). Mapping Women, Making Politics: Feminist Perspectives on Political Geography. London: Routledge.

Taylor P. (1989). Children and politics. Political Geography Quarterly, 8(1): 5-6.

Templeton T.S. (2020). 'That street is taking us to home': young children's photographs of public spaces. Children's Geographies, 18(1): 1-15. DOI: 10.1080/14733285.2018.1550573

Tulumello S., Cotella G., Othengrafen F. (2019). Spatial planning and territorial governance in Southern Europe between economic crisis and austerity. International Planning Studies, 25(1): 72-87. DOI: 1037//0033-2909.I26.1.78

Valentine G. (1996a). Angels and devils: moral landscapes of childhood. Environment and Planning D: Society and Space, 14: 581-599. DOI: 10.1068/d140581

Ead. (1996b). Children should be seen and not heard: The production and transgression of adults' public space. Urban Geography, 17(3): 205-220. DOI: 10.2747/02723638.17.3.205

Ead., Holloway S., a cura di (2000). Children's Geographies: Playing, living, learning. London e New York: Routledge.

van Lanen S. (2020). Exclusion and sense of displacement under austerity. Experiences from young adults in Ballymun, Dublin. ACME: An International E-Journal for Critical Geographies, 19(1): 352-363.

Ward C. (1978). The Child in the city. London: The Architectural Press.

Wodak R., Meyer M., a cura di (2009). Methods for Critical Discourse Analysis. London: SAGE Publications.

Wood B.E. (2012). Crafted within liminal spaces: Young people's everyday politics. Political Geography, 31(6): 337-346. DOI: 10.1016/j.polgeo.2012.05.003 